\title{
Granulomatous skin disease with a histological pattern of palisading granuloma - an atypical facial necrobiosis lipoidica or more?
}

\author{
Anna Rosińska-Więckowicz, Monika Bowszyc-Dmochowska \\ Department of Dermatology, Poznan University of Medical Sciences, Poznan, Poland \\ Adv Dermatol Allergol 2017; XXXIV (6): 618-621 \\ DOI: https://doi.org/10.5114/ada.2017.72468
}

\begin{abstract}
A 56-year-old woman was admitted to the Department of Dermatology due to the presence of disseminated patches on the skin of the face, and some sharply demarcated atrophic plaques on the skin of the right forearm and her left shin. Initially, the skin lesions had appeared on the left temple ca. 15 years ago, reportedly after a prolonged sun exposure. According to the patient, the lesions were erythematous, annular and non-itchy. Three years later, the aforementioned patches had appeared on the right half of the face, covering the skin of the temple, cheek and the nose. Seasonal aggravation of the skin symptoms during the summer months, as well as gradual resolution of the lesions during the autumn and the winter had been observed. Furthermore, a small asymptomatic patch had appeared on the dorsal side of the right shin ca. 10 years ago, and had been enlarging gradually throughout the years. Eventually, these lesions progressed to a sharply demarcated, yellow-brown atrophic plaques (Figure 1).
\end{abstract}

The patient's medical history included hypertension, treated with $\beta$-blockers, angiotensin converting enzyme inhibitor (ACEI), salicylic acid and proton-pump inhibitors (PPI). The patient also reported occasional arthritis of peripheral joints with tenderness and swelling. Rheumatoid arthritis was excluded based on the laboratory findings and $X$-ray examination.

In 2012, the patient underwent hospitalization at the dermatological ward of the district hospital. Biopsy material from the right arm skin lesion was taken for analysis purposes. Histological examination revealed a vacuolation of the basal epidermis, plus an inflammatory infiltration around the superficial and deep vessels in the dermis, as well as around the hair follicles. According to the pathologist, the histological pattern implied lupus erythematosus, however the finding was not evident. Indirect immunofluorescence of the serum did not reveal the presence of anti-nuclear antibodies (ANA). Based on the presence of discoid skin lesions, both on the face and the forearm, occasional arthritis, aggravation after the previous sun exposure, leukopenia $\left(3.2-3.76 \times 10^{3} / \mu \mathrm{l}\right)$ and the result of the skin biopsy, discoid lupus erythematosus was diagnosed. The treatment with antimalarial medications (hydroxychloroquine) was prescribed and avoidance of sun exposure combined with everyday use of SPF creams was also recommended.

On the day of admission, the aforesaid sharply demarcated patches located on the left temple, zygomatic arch and the cheek, as well as the nasolabial fold and the nose were present. The majority of the patches were annular, red, active on the border and with visible clearing in the center, plus slightly elevated on palpation. Moreover, on the flexural side of the right forearm, an ovoidal, ca. $4 \mathrm{~cm}$ in diameter, slightly pink, sharply demarcated and atrophic on palpation lesion was observed. Finally, on the extensor site of the right shin, a sharply demarcated, $7 \times 15 \mathrm{~cm}$ in dimension, yellow-brown atrophic plaque with telangiectasia was observed. Laboratory findings revealed a slightly elevated level of fasting blood glucose $(106 \mathrm{mg} / \mathrm{dl}$ and $101 \mathrm{mg} / \mathrm{dl})$, however the oral glucose tolerance test was normal. Direct immunofluorescence of the patient's serum revealed the presence of ANA, titer $1: 320$, granular fluorescence (fixed Hep-2 line cells). Endonuclear antibodies (Pm-Scl) were slightly positive. The patient was consulted by an ophthalmologist, who diagnosed the patient with hypertensive I/II stage angiopathy. Several skin scrapings were taken from the lesions present on the face, both for performing direct microscopy and for culturing. The cultured fragments of the skin scrapings taken from the lesions located on the left temple and cheek were found to be positive and the growth of dermatophyte, Trichophyton tonsurans was detected. Direct immunofluorescence of the biopsy material

Address for correspondence: Anna Rosińska-Więckowicz MD, PhD, Department of Dermatology, Poznan University of Medical Sciences, 49 Przybyszewskiego St, 60-355 Poznan, Poland, phone: +48 61869 1366, e-mail: rosinska.anna@gmail.com Received: 4.04.2016, accepted: 28.08.2016. 

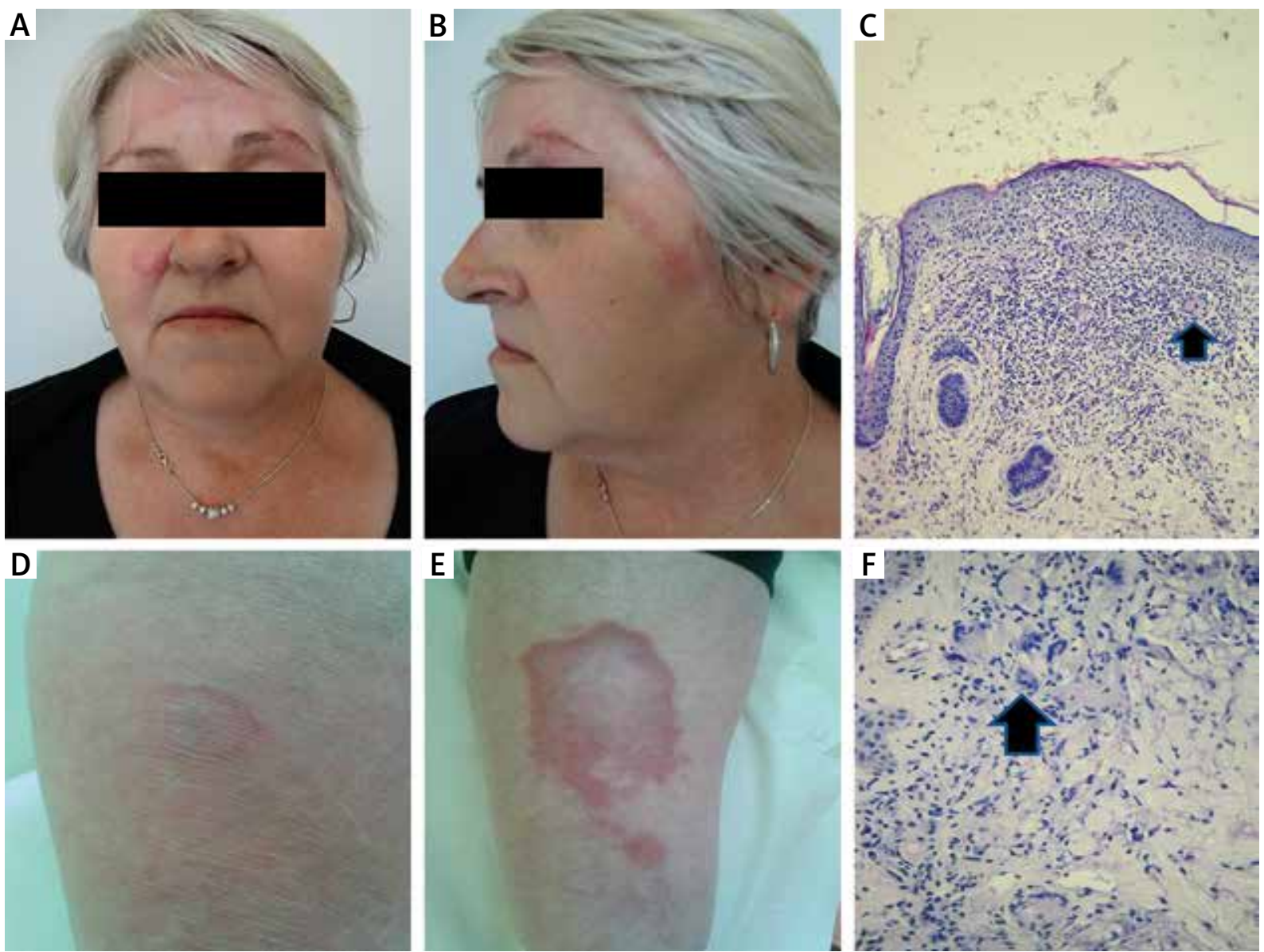

Figure 1. A, B - Erythematous plaques on the face, C - histopathological picture of the biopsy from the right cheek: focused but poorly-demarcated sub-epidermal inflammatory infiltrate with plasma cells, lymphocytes penetrating into the epidermis without necrobiosis but with ocasional giant cells (arrow), D - atrophic ovoid lesion on the flexural side of the right forearm, $\mathrm{E}$ - sharply demarcated atrophic plaque on the extensoral side of the right shin, $\mathrm{F}$ - histopathological picture of the biopsy from the lesion on the shin: fragment of the granuloma with necrobiotic collagen on the right and palisade of histiocytes and lymphocytes with giant cells (arrow) on the left

from the lesional skin of the face and the flexural side of the wrist (Lupus band test) did not confirm the presence of IgA, IgM, IgG deposits and the C3 complement component. Histological examination of the biopsy material taken from the right cheek revealed focused inflammatory infiltration with histiocytes, plasma cells and lymphocytes, penetrating into the thin epidermis with scattered giant cells (Figure $1 \mathrm{C}$ ). The necrobiosis or necrosis were absent. The description is consistent with chronic superficial nonspeciffic granulomatous dermatitis. Conversely, skin biopsy material from the border of the plaque on the right shin revealed the presence of atrophic epidermis, and the dermis with vascular thickening and areas of collagen degeneration (necrobiosis) with mucin strands, surrounded by inflammatory infiltration with histiocytes, lymphocytes and giant cells. According to the pathologist, the histological pattern represented typical signs of so called 'palisading granuloma', which may be observed in the course of necrobiosis lipoidica diabeticorum, granuloma annulare or granuloma disciformis (Miescher) (lat. granulomatosis disciformis chronica et progressiva). The clinical, histopathological features and the presence of typical lesion on the shin raised the suspicion of atypical facial necrobiosis lipoidica. Due to the presence of the left temple skin lesions, caused by infection with T. tonsurans, systemic treatment with terbinafine $250 \mathrm{mg}$ once a day for 14 days was administered. In order to treat the remaining skin lesions on the face, forearm and the shin, methylprednisolone aceponate $0.1 \%$ cream once a day and dapsone $50 \mathrm{mg}$ once a day were also prescribed. Four weeks after the patient's release from the hospital, a complete resolution of skin lesions on the left side of the face was observed. Conversely, skin lesions on the right side of the face were less infiltrated and erythematous, but nevertheless still present. The patient also reported that she felt generally unwell due to using dapsone. Although blood levels of methemoglobin during the treatment were normal, we have decided to dis- 
continue therapy with dapsone. Instead, treatment with tacrolimus monohydrate $0.1 \%$ twice a day for 4 weeks, thereafter once a day for 4 weeks was administered. The treatment resulted in a complete resolution of facial skin lesions, however no improvement on the forearm and the shin was observed. Dapsone and tacrolimus monohydrate $0.1 \%$ treatments were not tolerated well. Therefore, the patient decided not to undergo other forms of systemic treatments (UVA1, PUVA and cyclosporine A).

Little is known about etiology of granulomatous skin diseases, however recent studies revealed a critical role of tumor necrosis factor $\alpha$ (TNF- $\alpha$ ) in necrobiosis lipoidi$\mathrm{ca}(\mathrm{NL})$ and granuloma annulare (GA), as concentrations in sera of patients with these conditions are significantly higher than in healthy controls [1, 2]. Based on the clinical appearance of skin lesions, differential diagnostics of granulomatous skin diseases includes first GA, NL, Miescher granuloma (MG), rheumatoid nodule (RN), necrobiotic xanthogranuloma (NX), interstitial granulomatous dermatitis (IGD) and palisading neutrophilic and granulomatous dermatitis (PNGD). In addition, diseases such as actinic keratosis and lupus erythematosus (particularly in severely sun-exposed skin areas), localized scleroderma, tuberculosis, sarcoidosis and syphilis should also be taken into consideration [3]. Gradual appearance of clinical symptoms and longstanding course of granulomatous skin disorders may hinder rapid recognition of the disease, as it was observed in our patient. Initially, discoid lupus erythematosus (DLE) was diagnosed in our patient as she matched some of the criteria required by the American College of Rheumatology [2], while a few years later she developed skin lesions with clinical appearance of NL on extremities and lesions on the face resembling annular elastolytic giant cell granuloma (AEGCG). Moreover, granulomatous skin diseases or various histological granulomatous patterns, may coexist with each other, though presenting a different clinical picture, depending on the area of the affected skin, which was also observed in our patient [1]. While the atrophic plaque on the right shin and the atrophic ovoid lesion on the right forearm presented typical clinical pattern of NL, facial lesions required more careful diagnostic approach (DLE, AEGCG, NL, sarcoidosis).

Palisading granuloma (known also as necrotizing granuloma) is a histological variety of a granuloma, characterized by the presence of macrophages and giant cells arranged in a tier-like fashion on the border of collagen degeneration (necrobiosis) [4, 5]. The histological picture of palisading granuloma may be observed in the course of NL, GA, MG, RN, NX, IGD and PNGD [3-7]. Necrobiosis lipoidica presents itself with sharply demarcated, shiny yellow-brown atrophic plaques with telangiectasia, usually bilaterally on the pretibial area [4, 5]. It is estimated that $40 \%$ of patients with NL suffers from diabetes, and $15 \%$ present skin lesions in other parts of the body, such as the face, scalp, trunk, and upper extremities, where the diagnosis is more likely to be missed [3-8]. Necrobiosis lipoidica may precede the onset of diabetes mellitus in $15 \%$ of patients, however the presence or progression of the disease does not correlate with how well diabetes is controlled. In $75 \%$ of cases, lesions may become painless due to cutaneous nerve damage, but in $25 \%$ of patients they may be extremely painful [3-8]. Moreover, both $\mathrm{NL}$ and GA are related with Koebner phenomenon, which explains the common appearance of lesions at the site of trauma [8]. From the histopathological point of view, NL manifests with interstitial and palisaded granulomas that involve the subcutaneous tissue and dermis. The granulomas are arranged in a layered fashion with areas of collagen degeneration. Depletion of intradermal nerves and thickening of the blood vessel walls, as well as endothelial cell swelling found in the middle to deep dermis may also be observed. Although macrophages and giant cells are usually arranged in palisades on the border of necrobiosis, in some cases, non-caesatin granulomas may be observed - with numerous giant cells and relatively minor necrosis [1, 2, 4, 5]. The facial variety of NL is also worth consideration in differential diagnosis of patches and plaques in this area, especially if the typical lesions in typical localisation are present. The histopathology of the atypical facial necrobiosis lipoidica differs from the classic type of $\mathrm{NL}$ : there is dense granulomatous infiltrate with irregular distribution with conspicuous giant cells without necrobiosis or palisaded arangement, like in presented case. Contrary to the classic NL they also tend to resolve without scarring [9]. AEGCG manifests with red-brown or pink plaques with annular arrangement, observed in sun-exposed areas, such as the face and neck in adults [2]. Most patients report aggravation of AEGCG after sun exposure. In patients with no other signs of NL, diagnosis of AEGCG based exclusively on the clinical picture may be very difficult and the biopsy is usually inevitable $[4,6]$. It should be emphasized that elastosis of the dermis in AEFCG, as a result of chronic sun exposure, may also be related to the patient's age, while collagen degeneration with the presence of giant cells may not be as expressive as in $\mathrm{NL}$ $[1,4,6]$. The clue for the diagnosis of AEGCG is elastophagocytosis [9].

As for MG, the term refers to plaques on the extensor side of lower legs with a clinical picture of NL, however with no coexistence of diabetes, and is sometimes misused as a synonym of annular elastolytic giant cell granuloma [3-5].

Various infectious agents are also responsible for the granulomatous reaction, especially long-lasting infections including mycobacterial or fungal. The one form is nodular granulomatous perifolliculitis called Majocchi's granuloma caused by dermatophytes including trichophyton tonsurans [9]. We did not find extensive perifollicular supurrative and granulomatous inflammation with fungal hype in both biopsies, but it is not excluded 
that the long-lasting tinea incognito can take a form of chronic superficial granulomatous inflammation. The treatment with terbinafine followed by dapsone and topical antiinflammatory agents was succesfull in clearing of infectious agent and granulomatous reaction.

It is highly recommended to use creams with high SPF if granulomatous skin lesions are present in sun exposed areas [1, 3, 4]. Steroids, used topically as creams and ointments or in an intralesional manner, are the most common agents. Although steroids lessen inflammation of the early lesion, they also have almost no influence on atrophic lesions [1, 3-5]. Alternatively, 0.1\% topical tacrolimus ointment may be applied, showing good results, also in the treatment of ulcerated $\mathrm{NL}[4,10]$. In some patients the treatment with pentoxifylline, aspirin or platelets inhibitors was effective, as these drugs improve rheological properties of the blood $[4,11]$. Some patients responded well to therapy with antimalarial drugs, or dapsone and clofazimine $[1,4,6,12,13]$. Patients with disseminated lesions responded well to phototherapy (UVA1 or PUVA) or treatment with cyclosporine A [14, 15]. Solitary lesions were also treated with success with the photodynamic therapy and pulsed dye laser $[16,17]$. Tumor necrosis factor $\alpha$ inhibitors, such as etanercept, adalimumab and infliximab were also shown to improve granulomatous skin disorders $[18,19]$.

\section{Conflict of interest}

The authors declare no conflict of interest.

\section{References}

1. Reid S, Ladizinski B, Lee K, et al. Update on necrobiosis lipoidica: a review of etiology, diagnosis and treatment options. J Am Acad Dermatol 2013; 69: 783-91.

2. Hochberg M. Updating the American College of Rheumatology revised criteria for the classification of systemic lupus erythematosus. Arthritis Rheum 1997; 40: 1725.

3. Thornsberry L, English J. Etiology, diagnosis, and therapeutic management of granuloma annulare: an update. Am J Clin Dermatol 2013; 14: 279-90.

4. Braun-Falco Dermatologia. Burgdorf WHC, Plewig G, Wolff HH, Landhaller M. (eds). Czelej, Lublin 2010; 568-78.

5. Travassos A, Soares-De-Almeida L. Residents' corner. DeRmpath \& Clinic: differential diagnosis in palisading non-infectious granulomas. Diagnosis: Case 1: Granuloma annulare. Case 2: Necrobiosis lipoidica. Eur J Dermatol 2014; 24: 139-40.

6. Ecker R, Puchalsky D. Facial necrobiosis lipoidica treated with hydroxychloroquine. J Am Acad Dermatol 2005; 52: 52.

7. Lever's Histopathology of the Skin. $10^{\text {th }}$ ed. Elder D (eds). Lippincott Williams \& Wilkinson, London 2007; 427-57.

8. Miller RA. The Koebner phenomenon. Int J Dermatol 1982; 21: 192-7.

9. Patterson JW. Weedon's Skin Pathology, Fourth edition. Churchill Livingston Elsevier 2016.

10. Clayton T, Harrison PV. Successful treatment of chronic ulcerated necrobiosis lipoidica with $0.1 \%$ topical tacrolimus ointment. Br J Dermatol 2005; 152: 581-2.
11. Rhodes E. Necrobiosis lipoidica treated with ticlopidine. Acta Derm Venereol 1986; 66: 458.

12. Durupt F, Dalle S, Debarbieux S, et al. Successful treatment of necrobiosis lipoidica with antimalarial agents. Arch Dermatol 2008; 144: 118-9.

13. Benedix F, Geyer A, Lichte V, et al. Response of ulcerated necrobiosis lipoidica to clofazimine. Acta Derm Venereol 2009; 89: 651-2.

14. Stanway A, Rademaker M, Newman P. Healing of severe ulcerative necrobiosis lipoidica with cyclosporin. Australas J Dermatol 2004; 45: 119-22.

15. Beattie PE, Dawe RS, Ibbotson SH, Ferguson J. UVA1 phototherapy for treatment of necrobiosis lipoidica. Clin Exp Dermatol 2006; 31: 235-8.

16. Heidenheim M, Jemec GB. Successful treatment of necrobiosis lipoidica diabeticorum with photodynamic therapy. Arch Dermatol 2006; 142: 1548-50.

17. Moreno-Arias G, Camps-Fresneda A. Necrobiosis lipoidica diabeticorum treated with the pulsed dye laser. J Cosmet Laser Ther 2001; 3: 143-6.

18. Zhang K, Quan L, Hsu S. Treatment of necrobiosis lipoidica with etanercept and adalimumab. Dermatol Online J 2009; 15: 12.

19. Kolde G, Muche JM, Schulze P, et al. Infliximab: a promising new treatment option for ulcerated necrobiosis lipoidica. Dermatology 2003; 206: 180. 